WEIF-45

\title{
NOISE PERFORMANCE OF SUBMICRON HEMT CHANNELS UNDER LOW POWER CONSUMPTION OPERATION
}

\author{
J. M. Miranda , H. Zirath ${ }^{\#+}$, M. García ${ }^{\star}$ J. L. Sebastián \\ * Dept. of Applied Physics III, Faculty of Physics, University Complutense of Madrid \\ \# Chalmers University of Technology, Department of Microelectronics, Sweden \\ + Ericsson Microwave System, Mölndal, Sweden \\ o Raytheon RF Components, Andover, MA, USA
}

\begin{abstract}
We have investigated the noise performance of HEMT devices for low noise operation with the aim of developing a noise model valid for low power biasing. Analytical expressions useful for CAD models have been derived for the calculation of the Pospieszalski gate and drain temperatures, and have been verified from near pinchoff conditions up to usual bias voltages. An overshoot in the drain temperature as a function of the drain voltage has been observed at low drain currents in deep submicron gate lenght devices.
\end{abstract}

\section{INTRODUCTION}

Optimum noise performance in HEMT devices may demand for bias currents that can be too large in low power consumption applications. A good tradeoff between power consumption and low noise operation can only be achieved in a design if a model valid for a wide range of bias currents is available.

Different approaches have been pursued in the noise modeling of HEMT devices [1]-[4]. Among them, the one proposed by Pospieszalski [3] has emerged as one of the most accurate and convenient HFET noise models for CAD techniques. This model needs the empirical calculation of two different temperatures: $T_{G}$, associated to the intrinsic gate to source resistance and $T_{D}$, associated to the drain resistance. $T_{G}$ has a weak influence on the final calculated noise parameters, and in fact it has been shown that this temperature can be set to the room temperature if the drain current is not too high [5], [6]. However, $T_{D}$ does exhibit a strong influence on the drain current.

The aim of this work is to investigate how $T_{G}$ and $T_{D}$ vary as a function of the applied bias under low drain current operation, and to present an unified expression able to predict the values of these temperatures in a wide range of bias currents and gate voltages. With this aim in mind, on wafer noise measurements of HEMT devices were performed in the range $2-26 \mathrm{GHz}$ and for bias conditions ranging from near pinchoff to high cutoff frequency operation. In addition, the accuracy of the Pospieszalski model has been tested at all the measured bias points.

\section{DEVICE MEASUREMENTS}

The measured transistors were designed at the University of Chalmers and fabricated at the Philips Limeil Labs. They are $0.15 \mu \mathrm{m}$ gatelength devices based on GaAs technology with AlGaAs/InGaAs channels. These devices feature a grounded source with a via hole that provides stabilization and facilitates broad band design. A minimum noise figure less of $1 \mathrm{~dB}$ was measured at $26 \mathrm{GHz}$. under optimum noise performance biasing, whereas a transconductance of $700 \mathrm{mS} / \mathrm{mm}$ and maximum oscillation frecuency of $200 \mathrm{GHz}$ were both obtained under maximum gain bias conditions. 
On wafer noise and $S$ parameter measurements were performed in the range 2-26 $\mathrm{GHz}$ by using a probe station with a network analyzer (HP8510) and an automated noise measurement system ATN NP5. A Transmission Reflection Line (TRL) calibration kit was especially designed and fabricated in the same wafer of the devices, with the aim of avoiding the inaccuracies derived from the influence of the terminal pads.

\section{NOISE SIMULATION}

The small signal equivalent circuit of the devices was extracted using an inhouse Labview program based on the cold FET method [6], [7]. In order to test the accuracy of the circuital model, an error function based on the discrepancies between the measured and the simulated $\mathbf{S}$ parameters was estimated [8]. Figure 1 shows the calculated errors in the simulation of the $S$ parameters. At the highest bias currents and low drain voltages the error reaches high values due the neglection in our model of the gate to source resistance. However, the model provides a reasonable fit even for negative drain voltages. In addition, the errors in the $\mathrm{S}$ parameters were below $3 \%$ at all the bias points at which the noise measurements were performed.

The Pospieszalski model was used to simulate the noise performance of the devices [9]. In order to test the accuracy of this model, an error function was defined and estimated in the noise calculations. This function accounts for the discrepancies between the measured and the simulated noise parameters and has been described elsewhere [10]. The temperatures $T_{D}$ and $T_{G}$ were initially extracted from the noise measurements and then tuned by direct optimization. Once the optimized values were calculated, a non linear curve fitting was made to obtain an analytical expression for both $T_{G}$ and $T_{D}$ as a function of the drain current density.

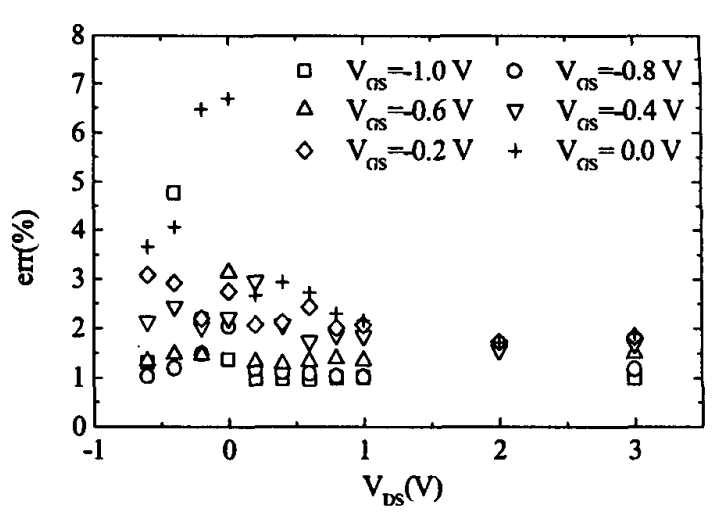

Figure 1. Errors in the extraction of the small signal parameters with the cold FET method. The drain voltages selected for the noise measurements were kept in the range $0.2 \mathrm{~V}-3 \mathrm{~V}$.

An accurate prediction of the noise parameters was achieved by assuming a linear dependence for $T_{G}$ and an hyperbolic one for $T_{D}$ with a current density offset,

$$
T_{D}=T_{D 0} \cosh \frac{J_{D}-J_{0}}{J_{1}}
$$

where $T_{D}$ is the drain temperature, and $T_{D 0}, J_{0}$ and $J_{1}$ are fitting factors. The final values of the fitting factors are shown in figure 2 for a 100 micron width transistor. This expression is able to reproduce two remarkable features of the drain temperature at low bias currents:

a) Despite the large values of the noise figure that can be obtained near pinchoff operation, $T_{D}$ tends to the room temperature when both the drain and gate voltages tend to zero. This feature demonstrates that $T_{D}$ behaves as a physical noise temperature: it tends to the room temperature near equilibrium.

b) $T_{D}$ exhibits an overshoot as a function of the drain voltage at low drain currents. This overshoot can clearly be observed in our samples at around $15 \mathrm{~mA}$ of drain current, where $T_{D}$ reaches values of near $2000 \mathrm{~K}$ for a $V_{D S}$ of $1.1 \mathrm{~V}$ 


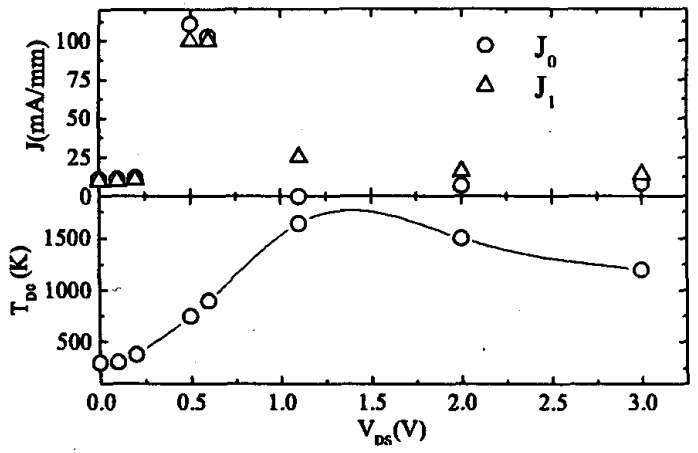

Figure 2. (a) Drain voltage dependence of the fitting parameters for the drain temperature.

and around $1300 \mathrm{~K}$ for $\mathrm{V}_{D S}$ equal to both $3 \mathrm{~V}$ and $0.5 \mathrm{~V}$. This effect could be attributed to the noise suppression under ballistic regime that has been observed by Matulionis et al. in pulsed noise measurements of ungated HEMT structures [11], [12].

Figure 3 shows the errors obtained in the calculation of the noise parameters before and after optimization, together with a typical fit of the $\mathrm{S}$ and noise parameters. The optimization could not improve the fitting at the highest drain voltages. However, below $1 \mathrm{~V}$ the calculated values of the gate temperatures became inaccurate and gave unrealistic values at the highest currents. The optimization in this case could help to obtain noticeable improvements in the accuracy of the calculations. It must be pointed out that once the temperatures are optimized the accuracy of the model is excellent near pinchoff operation, where noise figures in excess of $6 \mathrm{~dB}$ and noise resistances in excess of $350 \mathrm{Ohm}$ were measured.

\section{CONCLUSIONS}

The comparison between the measurements and simulation with the Pospieszalski model of the noise performance of HEMT devices have revealed us that this model can accurately reproduce the noise parameters of the device even under near pinchoff operation. Although at

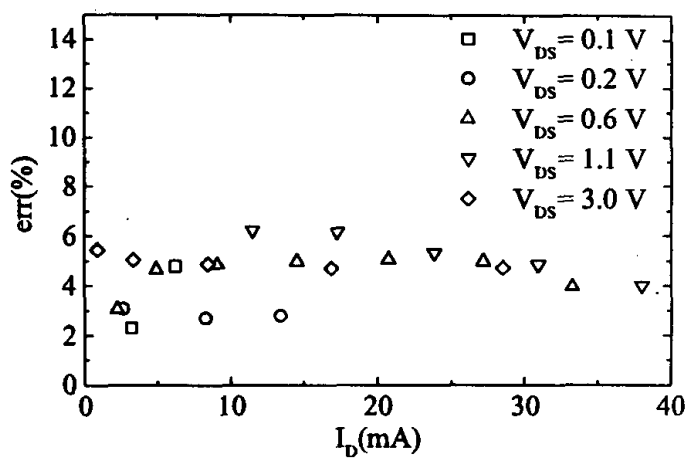

(a)

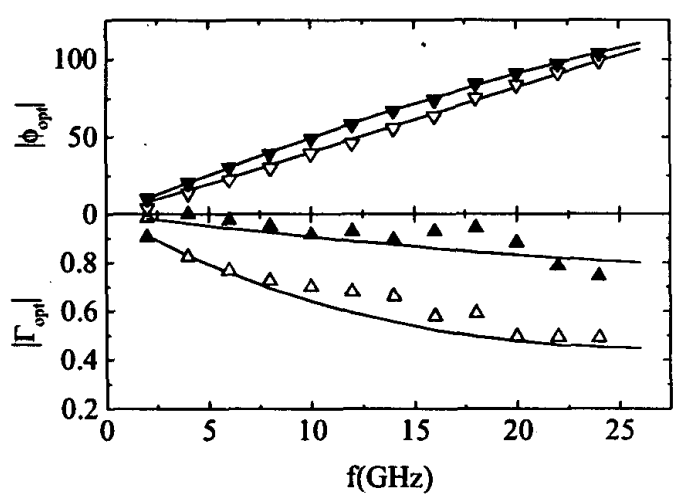

(b)

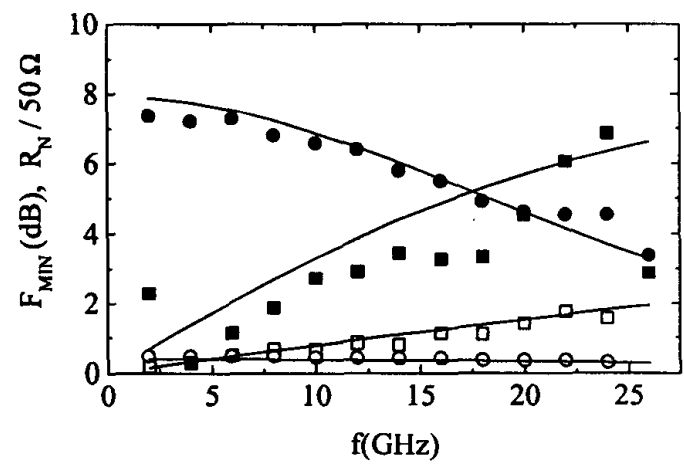

(c)

Figure 3. (a): Errors in the noise simulations after the optimization. (b), (c): Measured (symbols) and simulated (ines) noise parameters corresponding to $V_{D S}=3 V$ and $V_{G S}=0 \mathrm{~V}$ (open, error $=4 \%$ ) and $V_{D S}=0.1 \mathrm{~V}$ and $V_{G S}=-0.2$ $V$ (solid, error $=5 \%)$. Squares in (c) are $F_{M I N}$ and circles are normalized $R_{N}$. 
low drain bias voltages the direct extraction of the gate temperature from the measurements becomes inaccurate, a post optimization has enabled us to obtain a good accuracy in the predictions without the need for any additional refinement to the model. The optimized values of the gate and drain temperatures fit well to a linear increase for $T_{G}$ and an hyperbolic one for $T_{D}$. An overshoot in $T_{D}$ as a function of the gate voltage has been observed at low drain currents.

\section{ACKNOWLEDGEMENTS}

This work has partially been funded by the Swedish Foundation for Scientific Research through the project "Design of $60 \mathrm{GHz}$ WLANs". Paulius Sakalas, Christian Fager, and Ilcho Angelov are acknowledged for their valuable comments.

\section{REFERENCES}

[1] A. Cappy, "Noise Modeling and Measurement Techniques", IEEE Trans. on Microwave Theory and Techniques, 36 (1), pp. 1-10, 1988.

[2] F. Danneville, H. Happy, G. Dambrine, J. M. Belquin, A. Cappy, "Microscopic noise modeling and macroscopic noise models; How good a connection?" IEEE Trans. on Electron Devices, vol 41 no 5, pp. 779-786, 1994.

[3] M. W. Pospieszalski, "On the Measurement of Noise Parameters of Microwave Two-Port", IEEE Trans. on MTT, vol 34 no 4, pp. 256-458, 1986.

[4] R. A Pucel, H. A. Haus, H. Statz, "Signal and noise properties of gallium arsenide microwave field effect transistors", Advances in Electronics and Electron Physics, vol 38, New York Academic Press, 1975.

[5] P. Heymann, M. Rudolph, H. Prinzler, R. Doerner, L. Klapproth, G. Bök, "Experimental Evaluation of Microwave Field Effect Transistor Noise Models", IEEE Trans on MTT, vol 47 no 2, pp. 156-163, 1999.

[6] M. Garcia, J. Stenarson, K. Yhland, H. Zirath,"A New Extraction Method for the Two-Parameter FET Temperature Noise Model" IEEE Trans on MTT, vol 46 no 11, pp. 1679-1685, 1998.

[7] N. Rorsman, M. García, C. Karlsson and H. Zirath, "Accurate Small Signal Modeling of HFET's for Millimeter Wave Applications", IEEE Trans. on MTT, vol 44 no 3, pp. 432-437, 1996.

[8] M. García, N. Rorsman, K. Yhland, H. Zirath, I. Angelov, "Fast, Automatic and Accurate HFET SmallSignal Characterization", Microwave Journal, pp. 102117, July 1997.
[9] M. W. Pospieszalski, "Modeling of Noise Paramaters of MESFETs and MODFET and their Frequency and Temperature Dependence", IEEE Trans. on MTT, vol 37 no 9, pp. 1340-1350, 1989.

[10] J. M. Miranda, H. Zirath, J. L. Sebastián, "Noise Modeling of $0.15 \mu \mathrm{m}$ Gate Length HEMTs based on AlGaAs/InGaAs Channels", Proceedings of the GHz-2000 International Conference, Goteborg, Sweden, March 2000. [11] A.Matulionis, Hot-electron noise in HEMT channels and other 2-DEG structures (invited paper), in Proc. 5th European Gallium Arsenide and related III-V compounds Applications Symposium - GaAs'97, Bologna, Italy, pp. 165-174, September 3-5, 1997.

[12] V.Aninkevicius, B.Henle, E.Kohn, W.Leitch, J.Liberis, A.Matulionis, P.Sakalas, "Hot-electron noise in InGaAs - based channels", Proc. of 20th Workshop on Compound Semiconductor Devices and Integrated Circuits, Vilnius University Press, pp. 34-35, 1996. 